\title{
Commentary on: Considering Developing a Specialized Epilepsy Employment Program? The PEP Jobs Program Paradigm
}

\section{Robert T Fraser*}

Department of Rehabilitation Medicine, Neurology Vocational Services Unit, Epilepsy Center, University of Washington Seattle, WA, USA

The recently published editorial in Epilepsy and Behavior highlights the issues and concerns in developing a specialized program in neurological vocational rehabilitation. In this Commentary, diverse aspects of program development and maintenance will be synthesized from the Epilepsy and Behavior editorial and additional general considerations to be considered in specialized vocational rehabilitation program success are provided.

The unemployment rate in epilepsy is between two and three times the national average and may be as high as $50 \%$ for those with active seizures [1]. Specialized epilepsy vocational rehabilitation programs have historically had significantly better outcomes than state/federal programs, two to three times their level of success [2]. Unemployment for individuals in other neurological groups, such as those with moderate to severe TBI or MS similarly approximate or exceeds $50 \%$ and specialized vocational rehabilitation similarly do better as to placement outcome than traditionally available generic vocational rehabilitation programs $[3,4]$.

The benefits of successful employment for adults with neurological disabilities are numerous to include improved quality of life, medical coverage (generally), greater self-confidence, mood stability, increased social engagement, and positive effects on family functioning. Nevertheless, vocational rehabilitation services are generally lacking within our health care system due to factors such as lack of insurance coverage, short-term acute hospital stays, under-emphasis and lack of support by health care facilities, etc. Assistance with access to work, a "cornerstone life activity for adults," is often not available for individuals with neurological and other disabilities.

In order to remediate these vocational concerns, the development of specialized vocational rehabilitation programs certainly has its place. A summary of the program elements as presented in the Epilepsy and Behavior editorial (modeled on the San Francisco Bay Area PEP Jobs Program) include the following:

- Initial Program Drivers- In every case, there is a need for a "program driver" - an individual or individuals (e.g. physicians, Epilepsy Foundation affiliate board member, pharmaceutical or other concerned company, etc.) who recognize and are passionate about the need. There must be someone driving the need for funding and the provision of program infrastructure.

- Start-Up Funding- The actual amount of funding to be secured through a donor, grant sponsor, private company support, etc. will vary as to scope. It does, however, need to be sufficient for two-year program sustention in order to allow the procurement of diverse sources of long-term sustention.

- Patient Populations- Any vocational program needs clients and the available neurological patient populations needing the services must be apparent.

- Vocational Services Staffing- A key hiring consideration is finding quality personnel who are comfortable with and have experience in, servicing both the vocational services client and the employer. The hiring of certified rehabilitation counselors (CRCs) can be of benefit as they may be able to financially bill for more diversified services from the state vocational rehabilitation agency.

- Program Elements- A vocational rehabilitation program will involve client job preparatory activity and search services, employer development and direct placement/job retention services. Non-paid community based assessments (CBAs) can also be offered, with additional services being offered based upon staff expertise/CRC certification.

- Client Eligibility- A specialized vocational rehabilitation program can often accept individuals with more severe disabilities, excepting some cases where the disability is extreme (e.g. multiple weekly seizures, very marked cognitive impairment, etc.) There is always a delicate balance between funding sustention/services expansion and losing the important vocational services specialization.

- Vocational Evaluation Metrics- It is always important to be clear with funding sources about requested evaluation metrics. Typically, these will include the number of clients served and competitively placed, with 90 days or more retention information. Other metrics are generally specific to the funding source and can relate to discrete number of employer partners, number of successful non-paid client jobs tryouts, etc.

- Specialized Vocational Program Sustention- Program sustention considerations can often be overlooked or delayed. These deserve continuing focus from the program's initiation. The Bay Area PEP Jobs Program is exemplary in becoming a vendor for state vocational rehabilitation and Social Security's Ticket to Work program. It has also been successful in achieving continuing support from the Epilepsy Foundation of Northern California, medical foundations, pharmaceutical companies and private donors. Diversification of funding support is key here and the PEP program is outstanding in this area.

In the development of the new EFWorks program in Atlanta, a number of the PEP program elements were carefully taken into consideration. However, several additional elements received emphasis to include:

- Business Engagement- EFWorks was launched with a major marketing and education conference targeted to relevant area businesses and large non-profits. The development of a business advisory council was fostered by a volunteer commitment option as part of the launch's volunteer partnership agreement. Business

*Corresponding author: Robert T, Department of Rehabilitation Medicine, Neurology Vocational Services Unit, Epilepsy Center, University of Washington Seattle, WA, United States, E-mail: rfraser@uw.edu

Received April 18, 2018; Accepted April 28, 2018; Published April 30, 2018

Citation: Fraser RT (2018) Commentary on: Considering Developing a Specialized Epilepsy Employment Program? The PEP Jobs Program Paradigm. Int J Neurorehabilitation 5: 317. doi: 10.4172/2376-0281.1000317

Copyright: (c) 2018 Fraser RT. This is an open-access article distributed under the terms of the Creative Commons Attribution License, which permits unrestricted use, distribution, and reproduction in any medium, provided the original author and source are credited. 
Citation: Fraser RT (2018) Commentary on: Considering Developing a Specialized Epilepsy Employment Program? The PEP Jobs Program Paradigm. Int J Neurorehabilitation 5: 317. doi: 10.4172/2376-0281.1000317

engagement, however, requires innovative regular e-mail/other contact and periodic public relations events. Refinement of business engagement strategies is an ongoing process.

- Vocational Services Model- There are a number of models across vocational service delivery programs, e.g., the individualized placement model, supportive employment/enclaves, a "Place and Train" model, vocational case facilitation, a hybrid model, etc. The PEP program is individualized, while the Epilepsy Foundation of New England and EFWorks are considering a hybrid individualized and "Place and Train" model. Much of the direction is influenced by both the funding source and the nature of encouraging and available business partners and their needs.

The PEP specialized epilepsy vocational rehabilitation program's success at 10 years is truly exemplary as to key program elements being executed very well through the initial work of the all-important drivers, Dr. Peter Weber and Ken Laxer. EFWorks is currently building its model and the team is in the process of refining a manual for specialized program development that will be applicable, not only for adults with epilepsy, but also for those with other neurological disabilities. Outreach for input as to other helpful program elements and refinements are being conducted with other U.S. Epilepsy Foundation affiliates and specialized neurological vocational programs.

\section{References}

1. http://www.aesnet.org/clinical_resources/practice_tools/employment_resources

2. Fraser RT, Trejo W, Blanchard W (1984) Epilepsy rehabilitation: Evaluating specialized versus general agency outcome. Epilepsia 25: 332-337.

3. Wehman P, Kreutzer J, West MD, Sherron P, Zasler ND, et al. (1990) Return to work for persons with traumatic brain injury: A supported employment approach. Arch Phys Med Rehabil 71: 1047-1052.

4. Rumrill $P(2016)$ Return to work and job retention strategies for people with multiple sclerosis. In I. Schultz Handbook of return to work: From research to practice. Springer: New York, pp: 545-561. 